\title{
Effective Lagrangian for Nambu-Goldstone modes in nonequilibrium open systems
}

\author{
Masaru Hongo $\odot,{ }^{1,2,3}$ Suro Kim, ${ }^{4}$ Toshifumi Noumi, ${ }^{4,5}$ and Atsuhisa Ota $\oplus^{6}$ \\ ${ }^{1}$ Department of Physics, University of Illinois, Chicago, Illinois 60607, USA \\ ${ }^{2}$ RIKEN iTHEMS, RIKEN, Wako 351-0198, Japan \\ ${ }^{3}$ Research and Education Center for Natural Sciences, Keio University, Yokohama 223-8521, Japan \\ ${ }^{4}$ Department of Physics, Kobe University, Kobe 657-8501, Japan \\ ${ }^{5}$ Department of Physics, University of Wisconsin-Madison, Madison, Wisconsin 53706, USA \\ ${ }^{6}$ Department of Applied Mathematics and Theoretical Physics, University of Cambridge, \\ Cambridge CB3 OWA, United Kingdom
}

(Received 2 August 2019; revised 7 December 2020; accepted 4 January 2021; published 23 March 2021)

\begin{abstract}
We develop the effective field theory of diffusive Nambu-Goldstone (NG) modes associated with spontaneous internal symmetry breaking taking place in nonequilibrium open systems. The effective Lagrangian describing semiclassical dynamics of the NG modes is derived and matching conditions for low-energy coefficients are also investigated. Due to new terms peculiar to open systems, the associated NG modes show diffusive gapless behaviors in contrast to the propagating NG mode in closed systems. We demonstrate two typical situations relevant to the condensed matter physics and high-energy physics, where diffusive type-A or type-B NG modes appear.
\end{abstract}

DOI: 10.1103/PhysRevD.103.056020

\section{INTRODUCTION}

Symmetry and its realization give fundamental descriptions of physical systems from condensed matter physics to high-energy physics. Global symmetry of the system, if it exists, remains unbroken, or spontaneously broken in a given situation-e.g., in the ground state-and the resulting symmetry realization restricts a possible low-energy spectrum contained in the system: if spontaneous symmetry breaking (SSB) of continuous global symmetry takes place, it leads to the inevitable appearance of gapless excitations known as the Nambu-Goldstone (NG) modes according to the NG theorem [1-3] with a low-dimensional exception protected by the Mermin-Wagner theorem [4-6].

Although the original NG theorem is applicable to systems respecting the Lorentz symmetry in the ground state, the scope of its application has been just recently extended to several interesting directions. One example is a generalization of the $\mathrm{NG}$ theorem to a nonrelativistic system. In the absence of the Lorentz symmetry, there is generally a mismatch between the number of NG modes and the number of the broken symmetries, and the associated (so-called type-B) NG mode for broken internal

Published by the American Physical Society under the terms of the Creative Commons Attribution 4.0 International license. Further distribution of this work must maintain attribution to the author(s) and the published article's title, journal citation, and DOI. Funded by SCOAP ${ }^{3}$. symmetry often shows a quadratic dispersion relation $\omega=a \boldsymbol{k}^{2}(a \in \mathbb{R})$ [7-20]. Furthermore, the notion of symmetry and its spontaneous breaking are also extended to nonequilibrium closed systems [21-29] and open systems [30-33], where diffusive gapless modes appear. To achieve these developments, one most powerful tool, the effective field theory (EFT) [34-36], has been actively used. One can apply EFT to show a nonrelativistic generalization of the NG theorem $[8,15,17,18]$ and to describe nonequilibrium closed systems respecting conservation laws [21-29]. Dissipative effects in the low-energy spectrum are captured with the help of the Schwinger-Keldysh (real-time) formalism [37-39], and the doubled symmetry structure inherent in it plays a central role.

In this paper, taking one step further, we develop EFT for the NG modes resulting from spontaneous symmetry breaking in nonequilibrium open systems (or nonHermitian systems) where the conservation law is violated by considering e.g., the diffusive coupling between the system and environment [30-33]. Generalizing the CallanColeman-Wess-Zumino's (CCWZ) coset construction $[34,35]$ to the Schwinger-Keldysh formalism, we lay out a solid basis to construct the general effective Lagrangian for the open system NG modes, and apply it to two typical situations where the type-A and type-B NG modes appear.

\section{SYMMETRY STRUCTURE IN OPEN SYSTEMS}

Real-time dynamics of quantum systems can be systematically described by the use of the Schwinger-Keldysh 


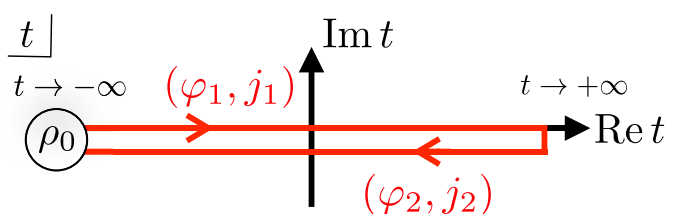

FIG. 1. Closed-time-path contour for the generating functional (1).

formalism [37-39]. One most basic quantity is the closedtime-path generating functional (CTPGF) defined by

$$
\begin{aligned}
Z\left[j_{1}, j_{2}\right] & \equiv \operatorname{Tr}\left(\hat{\rho}_{0} \hat{U}_{j_{2}}^{\dagger}(\infty,-\infty) \hat{U}_{j_{1}}(\infty,-\infty)\right) \\
& =\int \mathcal{D} \varphi_{1} \mathcal{D} \varphi_{2} \mathrm{e}^{\mathrm{i}\left(S\left[\varphi_{1} ; j_{1}\right]-S\left[\varphi_{2} ; j_{2}\right]\right)} \rho_{0}[\varphi],
\end{aligned}
$$

where $\hat{U}_{j}\left(t_{2}, t_{1}\right)$ denotes the time-evolution operator from $t_{1}$ to $t_{2}$ in the presence of the external field $j(t), \hat{\rho}_{0}$ the initial density operator at $t=-\infty$. In the second line, we used the path-integral expression for a system composed of dynamical field variables $\varphi$ with its action $S[\varphi ; j]$. One crucial point for the Schwinger-Keldysh formalism is the doubled degrees of freedom $\varphi \rightarrow\left\{\varphi_{1}, \varphi_{2}\right\}$ on the CTP (see Fig. 1). As a result, if the system originally enjoys $G$ symmetry, it is also doubled; the phase weight $e^{\mathrm{i}\left(S\left[\varphi_{1} ; j_{1}\right]-S\left[\varphi_{2} ; j_{2}\right]\right)}$ is invariant under $\left(G_{1} \times G_{2}\right)$ transformation acting on $\varphi_{1}$ and $\varphi_{2}$, respectively. ${ }^{1}$

The charges attached to that symmetry can be diffused if the system is put under the influence of environments, and, as a result, the above symmetry structure is modified. For example, let us consider the total system composed of two kinds of dynamical variables $\varphi=\{\phi, \sigma\}$ with system variables $\phi$ and environment variables $\sigma$. Then, the total action $S[\phi, \sigma ; j]$ is given by

$$
S[\phi, \sigma ; j]=S_{\mathrm{sys}}[\phi ; j]+S_{\mathrm{env}}[\sigma]+S_{\mathrm{int}}[\phi, \sigma],
$$

where $S_{\text {sys }}, S_{\text {env }}$ and $S_{\text {int }}$ are the actions of the system, environment and interaction part among them. Here, we assume that the background field is only coupled to the system variable $\phi$. By integrating out the environment variables, we define the influence functional $\Gamma\left[\phi_{1}, \phi_{2}\right]$ as [40]

$$
e^{\mathrm{i} \Gamma\left[\phi_{1}, \phi_{2}\right]} \equiv \int \mathcal{D} \sigma_{1} \mathcal{D} \sigma_{2} e^{\mathrm{i}\left(S_{\mathrm{env}}+S_{\text {int }}\right)} \rho_{0}[\phi, \sigma] .
$$

This procedure enables us to obtain the path-integral formula for open systems as

\footnotetext{
${ }^{1}$ However, note that nondiagonal part of $\left(G_{1} \times G_{2}\right)$ is explicitly broken due to the existence of the boundary, e.g., $\rho_{0}[\varphi]$. This broken symmetry is recently shown to be nonlinearly realized in the effective field theory for conserved systems such as dissipative hydrodynamics (see e.g., Refs. [24,27]).
}

$$
Z\left[j_{1}, j_{2}\right]=\int \mathcal{D} \phi_{1} \mathcal{D} \phi_{2} e^{\mathrm{i} S_{\text {open }}\left[\phi_{1}, \phi_{2} ; j_{1}, j_{2}\right]},
$$

where we defined the action for the open system $S_{\text {open }}$ :

$$
S_{\text {open }}=S_{\text {sys }}\left[\phi_{1} ; j_{1}\right]-S_{\text {sys }}\left[\phi_{2} ; j_{2}\right]+\Gamma\left[\phi_{1}, \phi_{2}\right] \text {. }
$$

The crucial point here is that the open system action $S_{\text {open }}\left[\phi_{1}, \phi_{2} ; j_{1}, j_{2}\right]$ is, in general, not invariant under the nondiagonal part of $\left(G_{1} \times G_{2}\right)$ transformation due to the presence of the influence functional $\Gamma\left[\phi_{1}, \phi_{2}\right]$. This is a manifestation of violating conservation laws. Even in that cases, the diagonal subgroup of doubled symmetry, which we call $G_{A}$ symmetry, still survives, and we can consider its spontaneous breaking in open quantum systems [30-33]. Our purpose is to construct the low-energy (IR) effective field theory describing such situations based on the underlying (UV) theory for open systems defined in Eq. (4).

Suppose that spontaneous $G_{A}$-symmetry breaking of the quantum open system takes place. Integrating out gapped degrees of freedom, we would like to develop the lowenergy EFT for the associated NG fields:

$$
Z\left[j_{1}, j_{2}\right]=\int \mathcal{D} \pi_{R} \mathcal{D} \pi_{A} e^{\mathrm{i} S_{\mathrm{eff}}\left[\pi_{R}, \pi_{A} ; j_{1}, j_{2}\right]}
$$

Here we introduced the effective action $S_{\text {eff }}=\int \mathrm{d}^{d} x \mathcal{L}_{\text {eff }}$ for $\left\{\pi_{R}, \pi_{A}\right\}-$ a combination of doubled NG fields in the socalled Keldysh basis whose properties will be elucidate in the next section. The vital point here is that we need to pay attention to several basic restrictions to the CTPGF (e.g., the probability conservation), which can be manifestly respected by demanding the following conditions for the effective action (see e.g., Refs. [24,27] in detail):

$$
\begin{gathered}
S_{\text {eff }}\left[\pi_{R}, \pi_{A}=0\right]=0, \\
S_{\text {eff }[}\left[\pi_{R}, \pi_{A}\right]^{*}=-S_{\text {eff }}\left[\pi_{R},-\pi_{A}\right], \\
\operatorname{Im} S_{\text {eff }}\left[\pi_{R}, \pi_{A}\right] \geq 0,
\end{gathered}
$$

where we switched off the external field. In the following, we will construct the effective action with vanishing external field $j$ based on the coset construction [34-36].

\section{COSET AND MAURER-CARTAN 1-FORM}

Let us first specify building blocks of the effective Lagrangian attached to spontaneous symmetry breaking of open systems $G_{A} \rightarrow H_{A}$ with unbroken symmetry subgroup $H_{A}$. In this paper, we employ the standard assumption in the coset construction that the broken generators are closed under the action of unbroken symmetry generators.

Naively speaking, it may be natural to identify the associated NG fields as a coordinate of the coset $G_{A} / H_{A}$ since the symmetry breaking pattern is $G_{A} \rightarrow H_{A}$. Nevertheless, 
due to the basic structure of the Schwinger-Keldysh EFT, we need to include the doubled NG fields into the effective Lagrangian. With the help of a set of NG fields $\boldsymbol{\pi}=\left(\pi_{R}, \pi_{A}\right)^{t}$, we then introduce doubled cosets $\xi_{i}(\boldsymbol{\pi}) \in$ $G_{i} / H_{i}(i=1,2)$, on which the $G_{i}$ transformation acts as

$$
\xi_{i}(\boldsymbol{\pi}) \stackrel{G_{i}}{\longrightarrow} g_{i} \xi_{i}(\boldsymbol{\pi}) h_{i}^{-1}\left(\boldsymbol{\pi}, g_{i}\right) \quad \text { with } \quad g_{i} \in G_{i}, \quad h_{i} \in H_{i} .
$$

Since we are considering the situation where all nondiagonal parts of $G_{1} \times G_{2}$ are explicitly broken in the open system, we only need to respect $G_{A}$ symmetry. By choosing a special representative

$$
\xi_{1,2}(\pi) \equiv \xi\left(\pi_{R}\right) e^{ \pm \mathrm{i} \pi_{A} / 2} \quad \text { with } \quad \xi\left(\pi_{R}\right) \equiv e^{\mathrm{i} \pi_{R}},
$$

which gives our definition of $\pi_{R}$ and $\pi_{A}$, we find the simple transformation rule under $(g, g) \in G_{A}$ transformation:

$$
\begin{aligned}
\xi\left(\pi_{R}\right) & \stackrel{G_{A}}{\longrightarrow} g \xi\left(\pi_{R}\right) h^{-1}\left(\pi_{R}, g\right), \\
\pi_{A} & \stackrel{G_{A}}{\longrightarrow} h\left(\pi_{R}, g\right) \pi_{A} h^{-1}\left(\pi_{R}, g\right) .
\end{aligned}
$$

In other words, we introduced the nonlinearly transforming NG field $\pi_{R}$ and the associated linearly transforming adjoint field $\pi_{A}$ under $G_{A}$ transformation. Note that $\pi_{R}$ and $\pi_{A}$ indeed parametrize the average and the difference parts of the doubled NG fields, which are required to satisfy the basic constraints (7a)-(7c).

With the help of the coset $\xi\left(\pi_{R}\right)$, we introduce the Maurer-Cartan 1-form as

$$
\alpha\left(\pi_{R}\right) \equiv \mathrm{i}^{-1} \xi^{-1}\left(\pi_{R}\right) \mathrm{d} \xi\left(\pi_{R}\right) .
$$

Then, projections of the Maurer-Cartan 1-form are shown to transform in the usual manner [34,35]:

$$
\begin{aligned}
& \alpha_{\perp}\left(\pi_{R}\right) \stackrel{G_{A}}{\longrightarrow} h \alpha_{\perp}\left(\pi_{R}\right) h^{-1}, \\
& \alpha_{\|}\left(\pi_{R}\right) \stackrel{G_{A}}{\longrightarrow} h \alpha_{\perp}\left(\pi_{R}\right) h^{-1}+\mathrm{i}^{-1} h \mathrm{~d} h^{-1},
\end{aligned}
$$

where we introduced projections onto broken and unbroken sectors as $\alpha_{\perp} \equiv \operatorname{tr}\left(X_{a} \alpha\right) X^{a}$ and $\alpha_{\|} \equiv \operatorname{tr}\left(X_{\alpha} \alpha\right) X^{\alpha}$. Among the generic generators $X_{I}$ of $G_{A}$ satisfying

$$
\begin{aligned}
{\left[X_{I}, X_{J}\right] } & =\mathrm{i} X_{K} f_{I J}^{K}, \\
\operatorname{tr}\left(X_{I} X_{J}\right) & \equiv g_{I J},
\end{aligned}
$$

we here introduced $X_{a}$ and $X_{\alpha}$ as generators which belong to broken and unbroken parts, respectively. ${ }^{2}$

\footnotetext{
${ }^{2}$ We will use the notation $I, J, \ldots$ to label generic generators of $G_{A}$, and $a, b, \ldots(\alpha, \beta, \ldots)$ for broken and (unbroken) ones.
}

In the following, assuming $g_{a \alpha}=0$, we lower (raise) the broken and unbroken indices by using the block diagonal part of the Cartan (inverse) metric $g_{a b}$ and $g_{\alpha \beta}\left(g^{a b}\right.$ and $\left.g^{\alpha \beta}\right)$.

Equation (12) tells us how derivatives of the MaurerCartan 1-form transform under the act of $G_{A}$. For instance, we can show a derivative of $\alpha_{\perp}$ defined by

$$
D_{\mu} \alpha_{\nu \perp} \equiv \partial_{\mu} \alpha_{\nu \perp}+\mathrm{i}\left[\alpha_{\mu \|}, \alpha_{\nu \perp}\right]
$$

transforms covariantly:

$$
D_{\mu} \alpha_{\nu \perp} \stackrel{G_{A}}{\longrightarrow} h D_{\mu} \alpha_{\nu \perp} h^{-1} .
$$

Transformation rules specified in Eqs. (10), (12), and (15) will be essential in constructing the effective Lagrangian of the NG mode in the subsequent section.

On the other hand, it seems unnecessary to examine the explicitly broken $\left(g, g^{-1}\right) \in G_{R}$-transformation property. Nevertheless, as will be shown later, we need them to clarify matching conditions for low-energy coefficients. Restricting ourselves to the semiclassical description of the NG fields, we obtain the following $G_{R}$-transformation rule (see the Appendix A for derivation):

$\pi_{R} \stackrel{G_{R}}{\longrightarrow} \pi_{R}+O\left(\hbar^{2}\right), \quad \pi_{A} \stackrel{G_{R}}{\longrightarrow} \pi_{A}+2\left[e^{-\mathrm{i} \pi_{R}} \theta e^{\mathrm{i} \pi_{R}}\right]_{\perp}+O\left(\hbar^{3}\right)$,

where we introduced $A_{\perp} \equiv \operatorname{tr}\left(X_{a} A\right) X^{a}$ with a transformation parameter $\theta \equiv \theta^{I} X_{I}$. Note that we count $\pi_{A}$ and $G_{R^{-}}$ transformation parameter as $O(\hbar)$ as is usual for the Schwinger-Keldysh EFT [24,27].

\section{EFFECTIVE LAGRANGIAN AND DIFFUSIVE NG MODES}

Taking account of basic constraints of the SchwingerKeldysh EFT (7a)-(7c) and $G_{A}$-transformation properties developed in the previous section, we now construct the general $G_{A}$-invariant effective Lagrangian by the use of the $\alpha_{\perp, \|}$ and $\pi_{A}$ as basic building blocks.

In this paper, we restrict ourselves to the semiclassical description of NG fields within second order in derivatives. In other words, we will collect possible terms up to $O\left(\pi_{A}^{2}, \partial_{t}^{2}, \partial_{i}^{2}\right)$. Here, we assume neither Lorentz nor Galilean symmetry but assume rotational symmetry because dissipative coupling to environment generally breaks the boost symmetry. As a result, temporal and spatial components (and derivatives) appear independently while the spatial component have to be contracted with each other using the Kronecker delta $\delta^{i j}$.

Now, let us write down all possible terms. First, we note that the condition (7) forces all terms to keep at least one $A$-type field $\pi_{A}$. This means that we have terms containing one $\pi_{A}$ or two $\pi_{A}$. Recalling the transformation rules given in Eqs. (10), (12), and (15), we find $O\left(\pi_{A}^{1}\right)$ six invariant terms as 
$O\left(\partial_{t}^{1}, \partial_{i}^{0}\right): \operatorname{tr}\left(\pi_{A} \alpha_{0 \perp}\right), \quad \operatorname{tr}\left(X_{I^{\prime}}\left[\pi_{A}, \alpha_{0 \perp}\right]\right)$,

$O\left(\partial_{t}^{2}, \partial_{i}^{0}\right): \operatorname{tr}\left(\pi_{A} D_{0} \alpha_{0 \perp}\right), \quad \operatorname{tr}\left(X_{I^{\prime}}\left[\pi_{A}, D_{0} \alpha_{0 \perp}\right]\right)$,

$O\left(\partial_{t}^{0}, \partial_{i}^{2}\right): \delta^{i j} \operatorname{tr}\left(\pi_{A} D_{i} \alpha_{j \perp}\right), \quad \delta^{i j} \operatorname{tr}\left(X_{I^{\prime}}\left[\pi_{A}, D_{i} \alpha_{j \perp}\right]\right)$,

where indices $I^{\prime}$ denote possible generators commuting with all the elements in $H_{A}:\left[X_{I^{\prime}}, X_{\alpha}\right]=0$. Note that rotational symmetry prohibits us to have $O\left(\partial_{i}^{1}\right)$ terms. Moreover, the leading-order term with two $A$-type field $\pi_{A}$ is given by

$$
O\left(\partial_{t}^{0}, \partial_{i}^{0}\right): \operatorname{tr}\left(\pi_{A} \pi_{A}\right) .
$$

Higher-derivative corrections could be incorporated in the similar manner as Ref. [41]. All the above terms in Eqs. (17) and (18) are all specified solely from the Lie algebra attached to broken and unbroken symmetries.

On the other hand, there are also other possible terms given by

$$
\operatorname{tr}\left(\pi_{A} \alpha_{0 \perp} \alpha_{0 \perp}\right), \quad \delta^{i j} \operatorname{tr}\left(\pi_{A} \alpha_{i \perp} \alpha_{j \perp}\right),
$$

and terms similar to the second terms in Eq. (17) but with anticommutator instead of commutator. Such terms generally contain the so-called $d$ coefficient $d_{I J K} \equiv \operatorname{tr}\left(X_{I}\left\{X_{J}, X_{K}\right\}\right)$, which depends on the representation of $G_{A}$ for microscopic constituents [42]. In that sense, we cannot specify these terms just from the symmetry breaking pattern $G_{A} / H_{A}$. Although consideration of these terms is interesting in its own right, ${ }^{3}$ we will omit these terms in the following analysis, leaving it as future works.

We are now ready to write down the semiclassical effective Lagrangian for NG mode in open systems. Summing up all terms in Eqs. (17) and (18), we find the leading-order $G_{A}$-invariant Schwinger-Keldysh effective Lagrangian is given by

$$
\begin{aligned}
\mathcal{L}_{\text {eff }}=-F[ & \frac{1}{f^{2}} \operatorname{tr}\left(\pi_{A} D_{0} \alpha_{0 \perp}\right)+\operatorname{itr}\left(m^{I^{\prime}} X_{I^{\prime}}\left[\pi_{A}, \alpha_{0 \perp}\right]\right) \\
& -\delta^{i j} \operatorname{tr}\left(\pi_{A} D_{i} \alpha_{j \perp}\right)+\gamma \operatorname{tr}\left(\pi_{A} \alpha_{0 \perp}\right) \\
& +i \operatorname{tr}\left(\zeta_{t}^{I^{\prime}} X_{I^{\prime}}\left[\pi_{A}, D_{0} \alpha_{0 \perp}\right]\right) \\
& \left.+\mathrm{i} \delta^{i j} \operatorname{tr}\left(\zeta_{S}^{I^{\prime}} X_{I^{\prime}}\left[\pi_{A}, D_{i} \alpha_{j \perp}\right]\right)-\mathrm{i} \frac{A}{2 F} \operatorname{tr}\left(\pi_{A} \pi_{A}\right)\right],
\end{aligned}
$$

where we used the covariant derivative of $\alpha_{\perp}$ defined in Eq. (14). We here rescaled the NG fields as $\pi_{R, A} \rightarrow \pi_{R, A} / F$, and assigned coefficients to make that of the third term to be unity. Note that all coefficients are assumed to be real in order to respect the Schwinger-Keldysh constraint (7b). A set of real parameters $\left\{f, m^{I^{\prime}}, \gamma, \zeta_{t}^{I^{\prime}}, \zeta_{s}^{I^{\prime}}, A\right\}$ gives low-energy coefficients, whose matching will be discussed shortly. The condition (7c) leads to $A \geq 0$, and when the charge is assumed to diffuse into the environment, we may also have $\gamma>0$. For notational simplicity, we also assumed that the broken symmetry generators are irreducible under the unbroken symmetry transformation $H$. If not, the EFT parameters $\left\{f, m^{I^{\prime}}, \gamma, \zeta_{t}^{I^{\prime}}, \zeta_{s}^{I^{\prime}}, A\right\}$ may have different values among irreducible sectors.

It should be emphasized that it corresponds to (or defines) type-A or type-B NG mode whether terms proportional to $m^{I^{\prime}}$ vanish or not [8-20]. Terms appearing in the second line of Eq. (20) are not invariant under $G_{R}$ transformation. Hence, they are peculiar to the Schwinger-Keldysh EFT for nonequilibrium open systems, where $G_{R}$ symmetry is explicitly broken. To see the quadratic part of the effective Lagrangian, expanding the Maurer-Cartan 1-form as $\alpha_{\mu \perp}=$ $\partial_{\mu} \pi_{R} / F+O\left(\pi_{R}^{2}\right)$ and $\alpha_{\mu \|}=O\left(\pi_{R}^{2}\right)$, we obtain

$$
\begin{aligned}
\mathcal{L}_{\text {eff }} & =-\rho_{a b} \pi_{A}^{a} \partial_{0} \pi_{R}^{b}+g_{a b}^{t} \partial_{0} \pi_{A}^{a} \partial_{0} \pi_{R}^{b}-g_{a b}^{s} \nabla \pi_{A}^{a} \cdot \nabla \pi_{R}^{b}+\frac{\mathrm{i}}{2} g_{a b}^{A} \pi_{A}^{a} \pi_{A}^{b} \\
& =\frac{\mathrm{i}}{2}\left(\begin{array}{cc}
\pi_{R}^{a} & \pi_{A}^{a}
\end{array}\right)\left(\begin{array}{cc}
0 & \mathrm{i}\left(g_{a b}^{t} \partial_{0}^{2}-\rho_{a b} \partial_{0}-g_{a b}^{s} \nabla^{2}\right) \\
\mathrm{i}\left(g_{a b}^{t} \partial_{0}^{2}+\rho_{a b} \partial_{0}-g_{a b}^{s} \nabla^{2}\right) & g_{a b}^{A}
\end{array}\right)\left(\begin{array}{c}
\pi_{R}^{b} \\
\pi_{A}^{b}
\end{array}\right),
\end{aligned}
$$

where we defined the following quantities:

$$
\begin{array}{ll}
\rho_{a b} \equiv-m^{I^{\prime}} f_{I^{\prime} a b}+\gamma g_{a b}, & g_{a b}^{A} \equiv A g_{a b}, \\
g_{a b}^{t} \equiv f^{-2} g_{a b}-\zeta_{t}^{I^{\prime}} f_{I^{\prime} a b}, & g_{a b}^{s} \equiv g_{a b}+\zeta_{s}^{I^{\prime}} f_{I^{\prime} a b},
\end{array}
$$

and neglected surface terms resulting from the integration by parts. We thus obtain the inverse retarded/advanced Green's functions for the NG modes as

\footnotetext{
${ }^{3}$ In particular, the second term in Eq. (19) gives an extension of a nonlinear coupling appearing in the Kardar-Parisi-Zhang equation [43], which is expected to control the universality class.
}

$$
\left(G_{R, A}^{a b}\right)^{-1}(\omega, \boldsymbol{k})=-g_{a b}^{t} \omega^{2} \mp \mathrm{i} \rho_{a b} \omega+g_{a b}^{s} \boldsymbol{k}^{2} .
$$

Solving $\operatorname{det}\left(G_{R}^{a b}\right)^{-1}(\omega, \boldsymbol{k})=0$ enables us to get the dispersion relation for the several types of NG modes depending on which parameters are present.

Figure 2 shows three cases of dispersion relations for the type-A and type-B NG modes with nonvanishing $\gamma$ and vanishing $\zeta$ terms. We see that all the NG modes show the diffusive behavior due to the nonvanishing negative imaginary part $\operatorname{Im} \omega(\boldsymbol{k})<0$. On the other hand, the real parts show qualitatively different behaviors between type-A and type-B modes; the type-A NG mode has no real part in the 
(a) Type-A NG mode: $(f, \gamma, m)=(1.0,1.0,0.0)$

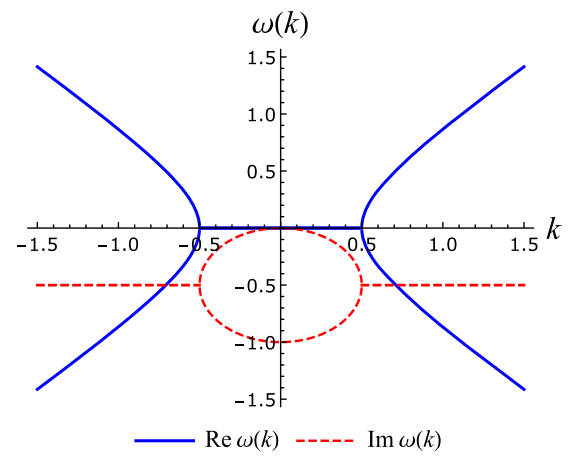

(b) Type-B NG mode: $(f, \gamma, m)=(1.0,1.0,0.2)$

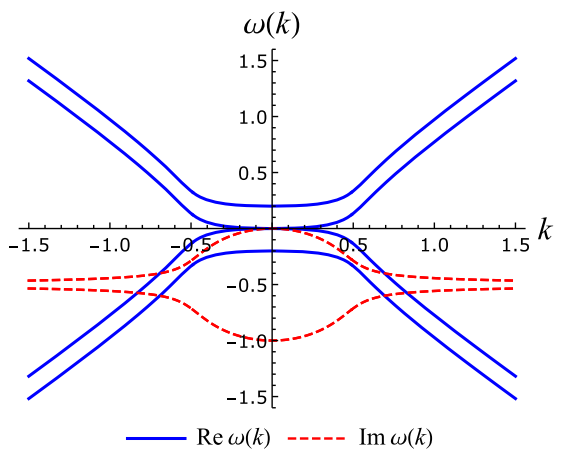

(c) Type-B NG mode: $(f, \gamma, m)=(1.0,1.0,0.5)$

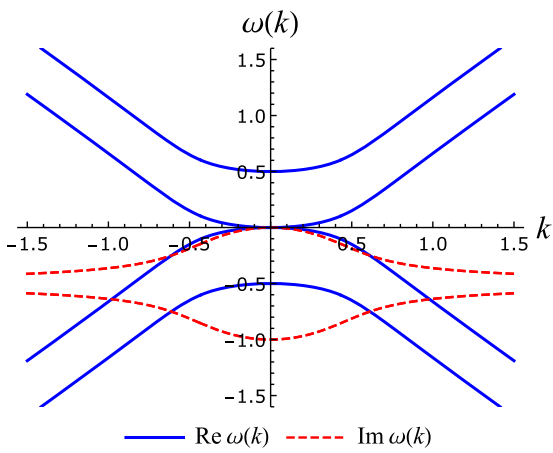

FIG. 2. Dispersion relations for (a) type-A NG mode and (b) and (c) type-B NG modes with vanishing $\zeta$ terms.

low-momentum region, while the type-B NG mode has that. Besides, the dispersion relation of the type-A NG mode has a special point $|\boldsymbol{k}|=f \gamma / 2$, beyond which it acquires a real part. This is in sharp contrast to the type-B NG mode because they do not have such a special point [compare Figs. 2(a), 2(b) and 2(c)]. The reason why the type-B NG mode does not have the special point is that its kinetic term is first order in time derivative and not negligible compared to the dissipation term even in the low-energy limit. It is worthwhile emphasizing that the presence of the special point for the type-A NG mode is a universal prediction following from our EFT although it depends on the model whether it really stays in the low-energy observable regime or not. More explicitly, its position in the momentum space depends on the value of both the condensate $f$ and diffusive coefficient $\gamma$. Figure 2 also demonstrates how the spectrum of type-A and type-B NG modes is connected by changing the parameter $m$ from $m=0$ [Fig. 2(a)] to $m \neq 0$ [Figs. 2(b) and 2(c)].

Perhaps, it is instructive to draw an analogy between the diffusive NG modes and damped harmonic oscillators. Indeed, the behavior shown in Fig. 2(a) allows a following simple interpretation: $|\boldsymbol{k}| \leq f \gamma / 2$ and $|\boldsymbol{k}| \geq f \gamma / 2$ for the type-A correspond to the overdamping and the underdamping, respectively, and the special point at $|\boldsymbol{k}|=f \gamma / 2$ corresponds to the critical damping. It is also possible to understand the behavior of the type-B NG mode in analogy to the damped harmonic oscillator. One can regard the typeB NG mode as an electrically charged damped harmonic oscillator in the two-spatial dimension under homogeneous background magnetic fields (applied perpendicular to the two-spatial direction).

Let us then specify the matching condition for lowenergy coefficients in $\rho_{a b}$ and $g_{a b}$ based on the linearized effective Lagrangian (21). In contrast to SSB in the ground state, we generally have new couplings peculiar to nonequilibrium open systems: $\rho_{a b}$ can have symmetric components whereas $g_{a b}^{t}$ and $g_{a b}^{s}$ can have antisymmetric components. To clarify the matching condition, noting that the leading infinitesimal transformation of the NG fields is given by $\pi_{R, A}^{b} \rightarrow \pi_{R, A}^{b}+\epsilon_{A, R}^{a}\left(\delta_{A_{a}, R_{a}} \pi_{R, A}^{b}+O(\epsilon, \pi)\right)$ with

$$
\begin{array}{ll}
\delta_{A_{a}} \pi_{R}^{b}=F \delta_{a}^{b}, & \delta_{A_{a}} \pi_{A}^{b}=0, \quad \text { and } \\
\delta_{R_{a}} \pi_{R}^{b}=0, & \delta_{R_{a}} \pi_{A}^{b}=F \delta_{b}^{a},
\end{array}
$$

we turn our attention to the leading part of Noether currents attached to the $G_{A, R}$ transformations:

$$
\begin{aligned}
& J_{a, A}^{\mu}=\left(\begin{array}{c}
F\left(g_{b a}^{t} \partial_{0} \pi_{A}^{b}-\rho_{b a} \pi_{A}^{b}\right) \\
-F g_{b a}^{s} \nabla \pi_{A}^{b}
\end{array}\right)+\cdots, \\
& J_{a, R}^{\mu}=\left(\begin{array}{c}
F\left(f^{-2} g_{a b} \partial_{0} \pi_{R}^{b}-m^{\alpha^{\prime}} f_{\alpha^{\prime} a b} \pi_{R}^{b}\right) \\
-F g_{a b} \nabla \pi_{R}^{b}
\end{array}\right)+\cdots,
\end{aligned}
$$

where the ellipses stand for higher-order terms with respect to $\pi_{R, A}$. Here $J_{a, R}^{\mu}$ is defined by the $G_{R}$-invariant part of the effective Lagrangian [corresponding to the first three terms in Eq. (20)], and hence, not conserved in open systems. Using these, we can identify the matching conditions, which are sorted into the first category also present in the closed system (see Appendix B for derivation)

$$
\begin{gathered}
\left.\left\langle\delta_{A_{a}} \pi_{R}^{b}\right\rangle\right|_{\pi=0}=F \delta_{a}^{b},\left.\quad\left\langle\delta_{R_{a}} J_{b, A}^{0}\right\rangle\right|_{\pi=0}=-F^{2} \rho_{a b}, \\
\left.G_{J_{a, A}^{0} J_{b, A}^{0}}(\omega, \boldsymbol{k}=0)\right|_{\omega=0}-\frac{F^{2}}{\omega} m^{I^{\prime}} f_{I^{\prime} a b}=\mathrm{i} \frac{F^{2}}{f^{2}} g_{a b},
\end{gathered}
$$

and the second one peculiar to the open system

$$
\begin{gathered}
\left.C_{a b}^{0}(\omega, \boldsymbol{k}=0)\right|_{\omega=0}=-\mathrm{i} F^{2} \gamma g_{a b}, \\
\left.\partial_{\omega} C_{a b}^{0}(\omega, \boldsymbol{k}=0)\right|_{\omega=0}=-F^{2} \zeta_{t}^{I^{\prime}} f_{I^{\prime} a b}, \\
\left.\partial_{k^{i}} C_{a b}^{i}(\omega=0, \boldsymbol{k})\right|_{k=0}=-F^{2} \zeta_{s}^{I^{\prime}} f_{I^{\prime} a b},
\end{gathered}
$$

where we defined

$$
\begin{aligned}
G_{J_{a, R}^{\mu} J_{b, A}^{\nu}}(\omega, \boldsymbol{k}) & \equiv \int \mathrm{d}^{d} x e^{\mathrm{i} \omega t-\mathrm{i} \boldsymbol{k} \cdot \boldsymbol{x}}\left\langle J_{a, R}^{\mu}(x) J_{b, A}^{\nu}(0)\right\rangle, \\
C_{a b}^{\nu}(\omega, \boldsymbol{k}) & \equiv \int \mathrm{d}^{d} x e^{\mathrm{i} \omega t-\mathrm{i} \boldsymbol{k} \cdot \boldsymbol{x}}\left\langle\partial_{\mu} J_{a, R}^{\mu}(x) J_{b, A}^{\nu}(0)\right\rangle .
\end{aligned}
$$


Here the angle bracket (with $\pi=0$ ) represents the path integral without dynamics of the NG fields. Note that $\gamma$ and $\zeta$ terms are matched by $C_{a b}^{\nu}(\omega, \boldsymbol{k})$ in the second category containing nonvanishing $\partial_{\mu} J_{a, R}^{\mu}(x)$ peculiar to open systems. While Eqs. (27) and (28) give matching conditions including $\zeta$ terms, we will consider two models with vanishing $\zeta$ terms in the following.

One remark on the matching condition is in order. In our formulation, the currents $J_{A, R}^{\mu}$ are defined in the IR EFT (20). While $J_{A}^{\mu}$ is equivalent to the UV theory one up to equations of motion for gapped UV modes that are integrated out, it is nontrivial to identify the UV operator equivalent to the IR current $J_{R}^{\mu}$ essentially because $G_{R}$ symmetry is explicitly broken. Also, the second condition in Eq. (27) involves a $G_{R}$ transformation. This might be again an obstruction to the UVIR matching since the equation of motion could not be $G_{R}$ invariant in open systems. Therefore, further studies are required for establishing the UV-IR matching conditions, leaving it for future works.

\section{EXAMPLES}

We here present examples for the type-A and type-B diffusive NG modes in Driven-dissipative Bose-Einstein condensate $(B E C)$ model and $b$. Dissipative $S U(2) \times U(1)$ linear sigma model.

a. Driven-dissipative BEC model.-As an example for a diffusive type-A NG mode, we consider a drivendissipative BEC system with $U(1)$ symmetry [31] whose Lagrangian reads

$$
\begin{aligned}
\mathcal{L}_{\text {open }}= & \phi_{A}^{\dagger}\left(\mathrm{i} \partial_{0}+\frac{\nabla^{2}}{2 m}+\mu+\mathrm{i} \kappa-\frac{g-\mathrm{i} \gamma_{0}}{2}\left|\phi_{R}\right|^{2}\right) \phi_{R}+(\text { H.c. }) \\
& +\frac{\mathrm{i}\left(A+2 \gamma_{0}\left|\phi_{R}\right|^{2}\right)}{2}\left|\phi_{A}\right|^{2}+O\left(\phi_{A}^{3}\right),
\end{aligned}
$$

where we introduced $\phi_{R} \equiv\left(\phi_{1}+\phi_{2}\right) / 2$ and $\phi_{A} \equiv \phi_{1}-\phi_{2}$ for the doubled Bosonic Schrödinger field. When $\kappa<0$ and $\gamma_{0}>0$, a driven-dissipative condensate $\bar{\phi}_{R}=v e^{-\mathrm{i} \omega_{0} t}$ could arise. Note that this solution is regarded as an example of time crystal in nonequilibrium open systems [30-33] since it spontaneously breaks a mixed part of time translation and $U(1)$ symmetry (see Appendix $\mathrm{C}$ for a detailed analysis on the driven-dissipative BEC model). In this case, terms proportional to $\zeta_{t}^{\alpha^{\prime}}, \zeta_{s}^{\alpha^{\prime}}$, and $m^{\alpha^{\prime}}$ all vanish, and the dispersion relation for the resulting NG modes is obtained as

$$
\omega=-\mathrm{i} \frac{g}{m \gamma_{0}} \boldsymbol{k}^{2}+O\left(\boldsymbol{k}^{4}\right) .
$$

We thus see that there exists a diffusive gapless mode, whose dispersion relation corresponds to the small $|k|\left(<k_{c}\right)$ part of Fig. 2 (a) with $k_{c}=f \gamma / 2$ (the exceptional point). Note that the number of the gapless modes agrees with that of broken symmetries; $\operatorname{rank}\left(g_{a b}\right)=\operatorname{dim} G_{A} / H_{A}$. b. Dissipative $S U(2) \times U(1)$ linear sigma model.-On the other hand, the dissipative $S U(2) \times U(1)$ model with a chemical potential defined by

$$
\begin{aligned}
\mathcal{L}_{\text {open }}= & \boldsymbol{\varphi}_{A}^{\dagger}\left[-\left(\partial_{0}+\mathrm{i} \mu\right)^{2}+\nabla^{2}-\gamma_{0} \partial_{0}-2 \lambda \boldsymbol{\varphi}_{R}^{\dagger} \boldsymbol{\varphi}_{R}\right] \boldsymbol{\varphi}_{R} \\
& +(\text { H.c. })+\mathrm{i} A \boldsymbol{\varphi}_{A}^{\dagger} \boldsymbol{\varphi}_{A}
\end{aligned}
$$

gives an example for the type-B NG mode [30]. This model can be regarded as an effective model describing the kaon condensation in the dense QCD matter $[9,10]$ coupled to the environment. Here $\varphi_{R, A}$ denote doubled two-component complex scalar fields. One can find a stationary solution for $\varphi_{R}$ 's equation of motion parametrized by e.g., $\overline{\boldsymbol{\varphi}}_{R}=(0, v)$ with $v=\mu / \sqrt{2 \lambda}$, which spontaneously breaks $G_{A}=S U(2) \times U(1)$ symmetry down to $H_{A}=$ $U(1)$. Due to a nonvanishing antisymmetric part of $\rho_{a b}$, this system contains the type-B NG mode and its gapped partner, whose dispersion relation is shown to be

$$
\begin{aligned}
& \omega=\frac{ \pm m-\mathrm{i} \gamma}{m^{2}+\gamma^{2}} \boldsymbol{k}^{2}+O\left(\boldsymbol{k}^{4}\right), \\
& \omega=( \pm m-\mathrm{i} \gamma) f^{2}+\frac{ \pm m+\mathrm{i} \gamma}{m^{2}+\gamma^{2}} \boldsymbol{k}^{2}+O\left(\boldsymbol{k}^{4}\right) .
\end{aligned}
$$

This gapless diffusive-propagating behavior of the type-B NG mode is shown in Fig. 2(b). Taking account of one diffusive type-A NG mode, we see that the total number of the gapless NG modes is smaller than that of broken symmetry, which is peculiar to type-B NG modes.

\section{SUMMARY AND DISCUSSION}

We have developed the EFT for the NG modes associated with SSB taking place in nonequilibrium open systems based on the coset construction. The derived effective Lagrangian enables us to describe the semiclassical dynamics of the diffusive NG modes, which can be applied to general open systems from open quantum system including non-Hermitian quantum systems to classical stochastic systems. As an application, we discussed the diffusive dispersion relation for type-A and type-B NG modes in two possible examples in condensed matter and high-energy physics.

Let us comment on some future problems. While we only investigate the dispersion relation of the diffusive NG modes with vanishing $\zeta_{t / s}$ terms, the constructed effective Lagrangian (20) contains much more information on manybody processes of the diffusive NG modes. It is interesting to investigate such information, e.g., low-energy theorems and possible instability caused by $\zeta_{t / s}$ terms based on our formalism. It would also be important to understand how the loop correction from NG fields affects the low-energy behavior of systems like the Mermin-Wagner theorem [4-6]. Also, it is worthwhile clarifying an additional constraint, known as dynamical Kubo-Martin-Schwinger/ 
thermal symmetry $[24,27,31,44,45]$ in thermal systems. Another interesting direction is to consider the SSB of spacetime symmetry in open systems. While the drivendissipative BEC discussed in this paper provides such a simplest example of time crystal, more general models may cause an instability towards the pattern formation $[46,47]$. We left these problems and wide applications of the developed EFT in many physical systems from cold atomic, condensed matter, high-energy, and active matter systems, as future works.

\section{ACKNOWLEDGMENTS}

The authors thank Yoshimasa Hidaka for critically useful discussions and invaluable comments. M. H. thanks Keisuke Fujii, Tetsuo Hatsuda and Yuki Minami for useful discussions. M. H. was supported by Japan Society of Promotion of Science (JSPS) Grant-in-Aid for Scientific Research (KAKENHI) Grant No. 18H01217, the Special Postdoctoral Researchers Program at RIKEN, and the U.S. Department of Energy, Office of Science, Office of Nuclear Physics under Award No. DE-FG0201ER41195. S. K. is supported in part by the Senshu Scholarship Foundation. T. N. is in part supported by JSPS KAKENHI Grants No. JP17H02894, No. JP18K13539, and No. 20H01902 and MEXT KAKENHI Grant No. JP18H04352. A. O. is supported by JSPS Overseas Research Fellowships. This work is partially supported by the Ministry of Education, Culture, Sports, Science, and Technology (MEXT)Supported Program for the Strategic Research Foundation at Private Universities "Topological Science" (Grant No. S1511006), and the RIKEN iTHEMS Program, in particular iTHEMS STAMP working group.

\section{APPENDIX A: DERIVATION OF $G_{R}$-TRANSFORMATION RULE (16)}

We here give the derivation of $G_{R}$-transformation rules of the NG fields given in Eq. (16). For that purpose, let us explicitly write the $G_{A}$ transformation given in Eq. (8) as

$$
\xi_{1,2}(\boldsymbol{\pi}) \rightarrow \xi_{1,2}\left(\boldsymbol{\pi}^{\prime}\right)=e^{ \pm \mathrm{i} \theta} e^{\mathrm{i} \pi_{R}} e^{ \pm \mathrm{i} \pi_{A} / 2} e^{-\mathrm{i} \beta_{1,2}(\boldsymbol{\pi}, \theta)},
$$

where $\theta=\theta^{I} X_{I}$ denotes the transformation parameter with the pullback $\beta(\boldsymbol{\pi}, \theta)=\beta^{\alpha}(\boldsymbol{\pi}, \theta) X_{\alpha}$. To determine the pullback $\beta(\boldsymbol{\pi}, \theta)$, based on the power counting scheme in which $\pi_{A}, \theta$ and $\beta_{1,2}$ are all counted as $O(\hbar)$, we expand $\xi_{1,2}(\pi)$ and their transformations (A1) as follows:

$$
\begin{aligned}
\xi_{1,2}(\boldsymbol{\pi})= & e^{\mathrm{i} \pi_{R}} \pm \frac{\mathrm{i}}{2} e^{\mathrm{i} \pi_{R}} \pi_{A}+O\left(\hbar^{2}\right) \\
\xi_{1,2}\left(\boldsymbol{\pi}^{\prime}\right)= & {\left[e^{\mathrm{i} \pi_{R}}+O\left(\hbar^{2}\right)\right] } \\
& \pm \frac{\mathrm{i}}{2} e^{\mathrm{i} \pi_{R}}\left[\pi_{A}+2 e^{-\mathrm{i} \pi_{R}} \theta e^{\mathrm{i} \pi_{R}} \mp 2 \beta_{1,2}+O\left(\hbar^{3}\right)\right] .
\end{aligned}
$$

Comparing these, we can express the transformation rules for $\pi_{R}$ and $\pi_{A}$ as

$$
\begin{aligned}
\pi_{R}^{\prime} & =\pi_{R}+O\left(\hbar^{2}\right), \\
\pi_{A}^{\prime} & =\pi_{A}+2 e^{-\mathrm{i} \pi_{R}} \theta e^{\mathrm{i} \pi_{R}}-2 \beta_{1}+O\left(\hbar^{3}\right) \\
& =\pi_{A}+2 e^{-\mathrm{i} \pi_{R}} \theta e^{\mathrm{i} \pi_{R}}+2 \beta_{2}+O\left(\hbar^{3}\right) .
\end{aligned}
$$

We therefore identify that the pullback $\beta_{1,2}$ takes the following form in our parametrization:

$$
\beta_{1}=-\beta_{2}=\left[e^{-\mathrm{i} \pi_{R}} \theta e^{\mathrm{i} \pi_{R}}\right]_{\|},
$$

where $A_{\|} \equiv \operatorname{tr}\left(X_{\alpha} A\right) X^{\alpha}$ is the unbroken component of $A$. This completes the semiclassical transformation rule of the NG fields given in Eq. (16).

\section{APPENDIX B: DERIVATION OF THE MATCHING CONDITION (27) and (28)}

Since the first two matching conditions in Eq. (27) immediately follow from the transformation rule (25), we here provide the derivation of the others in Eqs. (27) and (28). By using the retarded Green's function for NG modes given in Eq. (24), we can directly evaluate $G_{J_{a, R}^{0} J_{b, A}^{0}}(\omega, \boldsymbol{k})$ as follows:

$$
\begin{aligned}
G_{J_{a, R}^{0} J_{b, A}^{0}}(\omega, \boldsymbol{k}) & =\int \mathrm{d}^{d} x e^{\mathrm{i} \omega\left(t-t^{\prime}\right)-\mathrm{i} k \cdot\left(x-x^{\prime}\right)}\left\langle F\left(-\mathrm{i} \omega f^{-2} g_{a c}-m^{I^{\prime}} f_{I^{\prime} a c}\right) \pi_{R}^{c}(x) F\left(\mathrm{i} \omega g_{d b}^{t}-\rho_{d b}\right) \pi_{A}^{d}\left(x^{\prime}\right)\right\rangle \\
& =F^{2}\left(-\mathrm{i} \omega f^{-2} g_{a c}-m^{I^{\prime}} f_{I^{\prime} a c}\right)\left(-\mathrm{i} g^{t} \omega^{2}+\rho \omega+\mathrm{i} g^{s} \boldsymbol{k}^{2}\right)_{c d}^{-1}\left(\mathrm{i} \omega g_{d b}^{t}-\rho_{d b}\right) \\
& \stackrel{k \rightarrow 0}{\longrightarrow} F^{2}\left(\mathrm{i} \omega f^{-2} g_{a c}+m^{I^{\prime}} f_{I^{\prime} a c}\right)\left(\mathrm{i} \omega g^{t}-\rho\right)_{c d}^{-1}\left(\mathrm{i} \omega g_{d b}^{t}-\rho_{d b}\right) \frac{1}{\omega} \\
& =F^{2}\left(\mathrm{i}^{-2} g_{a b}+\omega^{-1} m^{I^{\prime}} f_{I^{\prime} a b}\right),
\end{aligned}
$$

which gives the matching condition for $f$, or the third equation in the matching condition (27).

We can show the remaining ones in Eq. (28) in the similar manner if we notice that the equation of motion for $\pi_{R}^{a}$ together with the definition of $J_{a, R}^{\mu}$ in Eq. (26) brings about 


$$
\partial_{\mu} J_{a, R}^{\mu}=F\left(\zeta_{t}^{I^{\prime}} f_{I^{\prime} a b} \partial_{0}^{2}-\gamma g_{a b} \partial_{0}+\zeta_{s}^{I^{\prime}} f_{I^{\prime} a b} \nabla^{2}\right) \pi_{R}^{b}+\mathrm{i} F g_{a b} \pi_{A}^{b} .
$$

This equation is a manifestation of the nonconserving feature of open systems. Then, we can evaluate the low-frequency/wave number behavior of $C_{a b}^{\nu}(\omega, \boldsymbol{k})$ in the similar manner with $G_{J_{a, R}^{0} J_{b, A}^{0}}(\omega, \boldsymbol{k})$, using the retarded Green's function of NG modes. Noting that $\left\langle\pi_{A}^{a}(x) \pi_{A}^{b}\left(x^{\prime}\right)\right\rangle=0$ thanks to the unitarity condition (7a), we can evaluate the time component $C_{a b}^{0}(\omega, \boldsymbol{k})$ as

$$
\begin{aligned}
C_{a b}^{0}(\omega, \boldsymbol{k}) & \equiv \int \mathrm{d}^{d} x e^{\mathrm{i} \omega\left(t-t^{\prime}\right)-\mathrm{i} k \cdot\left(\boldsymbol{x}-x^{\prime}\right)}\left\langle F\left(-\omega^{2} \zeta_{t}^{I^{\prime}} f_{I^{\prime} a c}+\mathrm{i} \omega \gamma g_{a c}-\boldsymbol{k}^{2} \zeta_{s}^{I^{\prime}} f_{I^{\prime} a c}\right) \pi_{R}^{c}(x) F\left(\mathrm{i} \omega g_{d b}^{t}-\rho_{d b}\right) \pi_{A}^{d}\left(x^{\prime}\right)\right\rangle \\
& =F^{2}\left(-\omega^{2} \zeta_{t}^{I^{\prime}} f_{I^{\prime} a c}+\mathrm{i} \omega \gamma g_{a c}-\boldsymbol{k}^{2} \zeta_{s}^{I^{\prime}} f_{I^{\prime} a c}\right)\left(-\mathrm{i} g^{t} \omega^{2}+\rho \omega+\mathrm{i} g^{s} \boldsymbol{k}^{2}\right)_{c d}^{-1}\left(\mathrm{i} \omega g_{d b}^{t}-\rho_{d b}\right) \\
& \stackrel{k \rightarrow 0}{\longrightarrow} F^{2}\left(-\omega^{2} \zeta_{t}^{I^{\prime}} f_{I^{\prime} a c}+\mathrm{i} \omega \gamma g_{a c}\right)\left(\mathrm{i} \omega g^{t}-\rho\right)_{c d}^{-1}\left(\mathrm{i} \omega g_{d b}^{t}-\rho_{d b}\right) \frac{-\mathrm{i}}{\mathrm{i} \omega} \\
& =F^{2}\left(\omega \zeta_{t}^{I^{\prime}} f_{I^{\prime} a b}-\mathrm{i} \gamma g_{a b}\right),
\end{aligned}
$$

whose $\omega \rightarrow 0$ limit and the derivative with respect to $\omega$ give the matching conditions for $\gamma$ and $\zeta_{t}$-the first two equations in Eq. (28). Also, the spatial component $C_{a b}^{i}(\omega, \boldsymbol{k})$ is similarly evaluated as

$$
\begin{aligned}
C_{a b}^{i}(\omega, \boldsymbol{k}) & \equiv \int \mathrm{d}^{d} x e^{\mathrm{i} \omega\left(t-t^{\prime}\right)-\mathrm{i} k \cdot\left(\boldsymbol{x}-\boldsymbol{x}^{\prime}\right)}\left\langle F\left(-\omega^{2} \zeta_{t}^{I^{\prime}} f_{I^{\prime} a c}+\mathrm{i} \omega \gamma g_{a c}-\boldsymbol{k}^{2} \zeta_{s}^{I^{\prime}} f_{I^{\prime} a c}\right) \pi_{R}^{c}(x) F\left(+\mathrm{i} k^{i}\right) g_{d b}^{s} \pi_{A}^{d}\left(x^{\prime}\right)\right\rangle \\
& =\mathrm{i} F^{2}\left(-\omega^{2} \zeta_{t}^{I^{\prime}} f_{I^{\prime} a c}+\mathrm{i} \omega \gamma g_{a c}-\boldsymbol{k}^{2} \zeta_{s}^{I^{\prime}} f_{I^{\prime} a c}\right) k^{i}\left(-\mathrm{i} g^{t} \omega^{2}+\rho \omega+\mathrm{i} g^{s} \boldsymbol{k}^{2}\right)_{c d}^{-1} g_{d b}^{s} \\
& \stackrel{\omega \rightarrow 0}{\longrightarrow}-F^{2} \zeta_{s}^{I^{\prime}} f_{I^{\prime} a b} k^{i},
\end{aligned}
$$

which gives the matching condition for $\zeta_{s}$-the third equation in Eq. (28).

\section{APPENDIX C: DIFFUSIVE TYPE-A NG MODE IN DRIVEN-DISSIPATIVE BOSE-EINSTEIN CONDENSATE}

We here provide a detailed analysis of the type-A NG mode followed by the presence of the driven-dissipative BoseEinstein condensate. Our starting point is the Schwinger-Keldysh Lagrangian

$$
\mathcal{L}_{\text {open }}=\left[\mathrm{i} \phi_{A}^{\dagger} \partial_{0} \phi_{R}-\frac{1}{2 m} \boldsymbol{\nabla} \phi_{A}^{\dagger} \mathbf{\nabla} \phi_{R}+\phi_{A}^{\dagger}\left(\mu+\mathrm{i} \kappa-\left(g-\mathrm{i} \gamma_{0}\right)\left|\phi_{R}\right|^{2}\right) \phi_{R}\right]+(\text { H.c. })+\frac{\mathrm{i}\left(A+4 \gamma_{0}\left|\phi_{R}\right|^{2}\right)}{2}\left|\phi_{A}\right|^{2},
$$

where we defined the Keldysh-basis fields $\phi_{R}=\left(\phi_{1}+\right.$ $\left.\phi_{2}\right) / 2$ and $\phi_{A} \equiv \phi_{1}-\phi_{2}$. We truncated the action at the quadratic order with respect to $A$-type field, which is regarded as the semiclassical approximation to open quantum systems described by the Lindblad equation (see, e.g., Ref. [31] for a review). Here (H.c.) represents the Hermitian conjugate, and $\kappa<0$ and $\gamma_{0}>0$ denote a driven particle-injection term and a dissipative term describing a nonlinear particle loss.

Using infinitesimal parameters $\epsilon$ for time-translational symmetry $\mathbb{R}_{t}$, and $\theta$ for internal $U(1)$ symmetry, we define infinitesimal $G_{A}$ and $G_{R}$ transformations in the semiclassical regime as

$$
\begin{aligned}
\delta_{A} \phi_{R} & =\epsilon_{A} \partial_{0} \phi_{R}+\mathrm{i} \theta_{A} \phi_{R}, \\
\delta_{A} \phi_{A} & =\epsilon_{A} \partial_{0} \phi_{A}+\mathrm{i} \theta_{A} \phi_{A}, \quad \text { and } \\
\delta_{R} \phi_{R} & =0, \\
\delta_{R} \phi_{A} & =\epsilon_{R} \partial_{0} \phi_{R}+\mathrm{i} \theta_{R} \phi_{R},
\end{aligned}
$$

where only $G_{A}$ symmetry is respected in Eq. (C1).

Let us then investigate a homogeneous symmetry breaking solution $\bar{\phi}_{R}$ and $\bar{\phi}_{A}$, on the top of which the type-A diffusive NG mode appears. Noting $\bar{\phi}_{A}=0$ resulting from the unitarity condition, we obtain the mean-field equation of motion for $\bar{\phi}_{R}$ :

$$
\left(\mathrm{i} \partial_{0}+\mu+\mathrm{i} \kappa-\left(g-\mathrm{i} \gamma_{0}\right)\left|\bar{\phi}_{R}\right|^{2}\right) \bar{\phi}_{R}=0 .
$$

The driven particle injection $\kappa<0$ makes the trivial solution $\bar{\phi}_{R}=0$ unstable, and as a result, there appears a nontrivial solution with a time-oscillating homogeneous condensate given by

$$
\bar{\phi}_{R}=v e^{-\mathrm{i} \omega_{0} t} \quad \text { with } \quad v^{2}=-\frac{\kappa}{\gamma_{0}}, \quad \omega_{0}=g v^{2}-\mu .
$$


Due to the time-oscillating condensate, this solution breaks $G_{A}=\mathbb{R}_{t} \times U(1)_{M}$ symmetry down to $H_{A}=U(1)_{M+t}$. Here $U(1)_{M+t}$ denotes the combination of the time translation and $U(1)$ transformation satisfying $\omega_{0} \epsilon_{A}-\theta_{A}=0$, which let the driven-dissipative condensate (C4) invariant.

Next, we consider the fluctuation on the top of the above symmetry breaking solution and derive the effective Lagrangian for the diffusive NG mode. The embedding of Nambu-Goldstone mode reads

$$
\begin{aligned}
& \phi_{R} \simeq e^{\mathrm{i} \pi_{R}-\mathrm{i} \omega_{0} t}\left[v+\sigma_{R}\right], \\
& \phi_{A} \simeq e^{\mathrm{i} \pi_{R}-\mathrm{i} \omega_{0} t}\left[\mathrm{i}\left(v+\sigma_{R}\right) \pi_{A}+\sigma_{A}\right],
\end{aligned}
$$

where $\pi_{R, A}$ and $\sigma_{R, A}$ represent a phase (Nambu-Goldstone) and gapped amplitude field, respectively. To see the semiclassical dynamics of those fields, we dropped the higher-order terms including more than two $A$-type fields. Substituting the embedding (C5) into Eq. (C1) and focusing on the leading-order quadratic terms, we obtain the following result:

$$
\begin{aligned}
& \mathcal{L}_{\text {open }}=-\frac{2 v^{2}}{2 m} \nabla \pi_{A} \nabla \pi_{R}+\frac{\mathrm{i} \bar{A} v^{2}}{2} \pi_{A}^{2} \\
& -\frac{1}{2}\left(\begin{array}{ll}
\sigma_{R} & \sigma_{A}
\end{array}\right)\left(\begin{array}{cc}
0 & -\frac{1}{m} \boldsymbol{\nabla}^{2}+4 g v^{2} \\
-\frac{1}{m} \nabla^{2}+4 g v^{2} & -\mathrm{i} \bar{A}
\end{array}\right)\left(\begin{array}{c}
\sigma_{R} \\
\sigma_{A}
\end{array}\right)+2 v\left(2 \gamma_{0} v^{2} \pi_{A}-\partial_{0} \pi_{A} \quad-\partial_{0} \pi_{R}\right)\left(\begin{array}{c}
\sigma_{R} \\
\sigma_{A}
\end{array}\right) .
\end{aligned}
$$

After integrating out the gapped mode $\sigma$ and taking the low-energy limit $\nabla^{2} / m \ll 4 g v^{2}$, we obtain the effective Lagrangian of the NG fields as

$$
\begin{aligned}
& \mathcal{L}_{\text {eff }}=-\frac{2 v^{2}}{2 m} \boldsymbol{\nabla} \pi_{A} \nabla \pi_{R}+\frac{\mathrm{i} \bar{A} v^{2}}{2} \pi_{A}^{2} \\
& -\frac{\mathrm{i}(2 \mathrm{i} v)^{2}}{2}\left(\begin{array}{ll}
2 \gamma_{0} v^{2} \pi_{A}-\partial_{0} \pi_{A} & -\partial_{0} \pi_{R}
\end{array}\right)\left(\begin{array}{cc}
0 & -\frac{\mathrm{i}}{m} \boldsymbol{\nabla}^{2}+4 \mathrm{i} g v^{2} \\
-\frac{\mathrm{i}}{m} \boldsymbol{\nabla}^{2}+4 \mathrm{i} g v^{2} & \bar{A}
\end{array}\right)^{-1}\left(\begin{array}{c}
2 \gamma_{0} v^{2} \pi_{A}-\partial_{0} \pi_{A} \\
-\partial_{0} \pi_{R}
\end{array}\right) \\
& \simeq-\frac{2 \gamma_{0} v^{2}}{g} \pi_{A} \partial_{0} \pi_{R}-\frac{2 v^{2}}{2 m} \nabla \pi_{A} \nabla \pi_{R}+\frac{\mathrm{i} \bar{A} v^{2}}{2}\left(1+\frac{\gamma_{0}^{2}}{g^{2}}\right) \pi_{A}^{2} \\
& =\frac{\mathrm{i}}{2}\left(\begin{array}{ll}
\pi_{R} & \pi_{A}
\end{array}\right)\left(\begin{array}{cc}
0 & \mathrm{i}\left(-\frac{\gamma_{0} v^{2}}{g} \partial_{0}-\frac{v^{2}}{m} \boldsymbol{\nabla}^{2}\right) \\
\mathrm{i}\left(\frac{\gamma_{0} v^{2}}{g} \partial_{0}-\frac{v^{2}}{m} \boldsymbol{\nabla}^{2}\right) & \bar{A} v^{2}\left(1+\frac{\gamma_{0}^{2}}{g^{2}}\right)
\end{array}\right)\left(\begin{array}{c}
\pi_{R} \\
\pi_{A}
\end{array}\right),
\end{aligned}
$$

where we introduced $\bar{A} \equiv A+4 \gamma_{0} v^{2}$. The derived effective Lagrangian provides the following inverse retarded Green's function in the Fourier space:

$$
G_{R}^{-1}(\omega, \boldsymbol{k})=-\mathrm{i} \frac{\gamma_{0} v^{2}}{g} \omega+\frac{v^{2}}{m} \boldsymbol{k}^{2}
$$

which results in the expected diffusive dispersion relation for the type-A NG mode

$$
\omega=-\mathrm{i} \frac{g}{m \gamma_{0}} \boldsymbol{k}^{2} .
$$

We can also specify the $G_{A, R}$ transformation rules of NG modes as

$$
\delta_{A} \pi_{R}=\epsilon_{A} \partial_{0} \pi_{R}-\omega_{0} \epsilon_{A}+\theta_{A}, \quad \delta_{A} \pi_{A}=\epsilon_{A} \partial_{0} \pi_{A}, \quad \text { and } \quad \delta_{R} \pi_{R}=0, \quad \delta_{R} \pi_{A}=\epsilon_{R} \partial_{0} \pi_{R}-\omega_{0} \epsilon_{R}+\theta_{R},
$$

from which one sees that $\pi_{R / A}$ nonlinearly transforms under the $G_{A / R}$ transformation while linearly transforms under the $G_{R / A}$ transformation. 
[1] Y. Nambu and G. Jona-Lasinio, Dynamical model of elementary particles based on an analogy with superconductivity. 1, Phys. Rev. 122, 345 (1961).

[2] J. Goldstone, Field theories with superconductor solutions, Nuovo Cimento 19, 154 (1961).

[3] J. Goldstone, A. Salam, and S. Weinberg, Broken symmetries, Phys. Rev. 127, 965 (1962).

[4] N. D. Mermin and H. Wagner, Absence of Ferromagnetism or Antiferromagnetism in One-Dimensional or TwoDimensional Isotropic Heisenberg Models, Phys. Rev. Lett. 17, 1133 (1966).

[5] P. C. Hohenberg, Existence of long-range order in one and two dimensions, Phys. Rev. 158, 383 (1967).

[6] S. R. Coleman, There are no Goldstone bosons in two dimensions, Commun. Math. Phys. 31, 259 (1973).

[7] H. B. Nielsen and S. Chadha, On how to count Goldstone bosons, Nucl. Phys. B105, 445 (1976).

[8] H. Leutwyler, Nonrelativistic effective Lagrangians, Phys. Rev. D 49, 3033 (1994).

[9] V. A. Miransky and I. A. Shovkovy, Spontaneous Symmetry Breaking with Abnormal Number of Nambu-Goldstone Bosons and Kaon Condensate, Phys. Rev. Lett. 88, 111601 (2002).

[10] T. Schafer, D. T. Son, M. A. Stephanov, D. Toublan, and J. J. M. Verbaarschot, Kaon condensation and Goldstone's theorem, Phys. Lett. B 522, 67 (2001).

[11] Y. Nambu, Spontaneous breaking of Lie and current algebras, J. Stat. Phys. 115, 7 (2004).

[12] T. Brauner, Spontaneous symmetry breaking and NambuGoldstone bosons in quantum many-body systems, Symmetry 2, 609 (2010).

[13] H. Watanabe and T. Brauner, Number of Nambu-Goldstone bosons and its relation to charge densities, Phys. Rev. D 84, 125013 (2011).

[14] Y. Hidaka, Counting Rule for Nambu-Goldstone Modes in Nonrelativistic Systems, Phys. Rev. Lett. 110, 091601 (2013).

[15] H. Watanabe and H. Murayama, Unified Description of Nambu-Goldstone Bosons without Lorentz Invariance, Phys. Rev. Lett. 108, 251602 (2012).

[16] A. Nicolis and F. Piazza, Implications of Relativity on Nonrelativistic Goldstone Theorems: Gapped Excitations at Finite Charge Density, Phys. Rev. Lett. 110, 011602 (2013); Erratum, Phys. Rev. Lett. 110, 039901 (2013).

[17] A. Nicolis, R. Penco, F. Piazza, and R. A. Rosen, More on gapped Goldstones at finite density: More gapped Goldstones, J. High Energy Phys. 11 (2013) 055.

[18] H. Watanabe and H. Murayama, Effective Lagrangian for Nonrelativistic Systems, Phys. Rev. X 4, 031057 (2014).

[19] D. A. Takahashi and M. Nitta, Counting rule of NambuGoldstone modes for internal and spacetime symmetries: Bogoliubov theory approach, Ann. Phys. (Amsterdam) 354, 101 (2015).

[20] T. Hayata and Y. Hidaka, Dispersion relations of NambuGoldstone modes at finite temperature and density, Phys. Rev. D 91, 056006 (2015).

[21] S. Endlich, A. Nicolis, R. A. Porto, and J. Wang, Dissipation in the effective field theory for hydrodynamics: First order effects, Phys. Rev. D 88, 105001 (2013).
[22] S. Grozdanov and J. Polonyi, Viscosity and dissipative hydrodynamics from effective field theory, Phys. Rev. D 91, 105031 (2015).

[23] F. M. Haehl, R. Loganayagam, and M. Rangamani, Adiabatic hydrodynamics: The eightfold way to dissipation, J. High Energy Phys. 05 (2015) 060.

[24] M. Crossley, P. Glorioso, and H. Liu, Effective field theory of dissipative fluids, J. High Energy Phys. 09 (2017) 095.

[25] F. M. Haehl, R. Loganayagam, and M. Rangamani, Topological sigma models and dissipative hydrodynamics, J. High Energy Phys. 04 (2016) 039.

[26] K. Jensen, N. Pinzani-Fokeeva, and A. Yarom, Dissipative hydrodynamics in superspace, J. High Energy Phys. 09 (2018) 127.

[27] P. Glorioso, M. Crossley, and H. Liu, Effective field theory of dissipative fluids (II): Classical limit, dynamical KMS symmetry and entropy current, J. High Energy Phys. 09 (2017) 096.

[28] F. M. Haehl, R. Loganayagam, and M. Rangamani, Effective action for relativistic hydrodynamics: Fluctuations, dissipation, and entropy inflow, J. High Energy Phys. 10 (2018) 194.

[29] K. Jensen, R. Marjieh, N. Pinzani-Fokeeva, and A. Yarom, A panoply of Schwinger-Keldysh transport, SciPost Phys. 5, 053 (2018).

[30] Y. Minami and Y. Hidaka, Spontaneous symmetry breaking and Nambu-Goldstone modes in dissipative systems, Phys. Rev. E 97, 012130 (2018).

[31] L. M. Sieberer, M. Buchhold, and S. Diehl, Keldysh field theory for driven open quantum systems, Rep. Prog. Phys. 79, 096001 (2016).

[32] M. Hongo, S. Kim, T. Noumi, and A. Ota, Effective field theory of time-translational symmetry breaking in nonequilibrium open system, J. High Energy Phys. 02 (2019) 131.

[33] T. Hayata and Y. Hidaka, Diffusive Nambu-Goldstone modes in quantum time crystals, arXiv:1808.07636.

[34] S. R. Coleman, J. Wess, and B. Zumino, Structure of phenomenological Lagrangians. 1, Phys. Rev. 177, 2239 (1969).

[35] C. G. Callan, Jr., S. R. Coleman, J. Wess, and B. Zumino, Structure of phenomenological Lagrangians. 2, Phys. Rev. 177, 2247 (1969).

[36] S. Weinberg, Phenomenological Lagrangians, Physica (Amsterdam) 96A, 327 (1979).

[37] J. S. Schwinger, Brownian motion of a quantum oscillator, J. Math. Phys. (N.Y.) 2, 407 (1961).

[38] L. V. Keldysh, Diagram technique for nonequilibrium processes, Zh. Eksp. Teor. Fiz. 47, 1515 (1964).

[39] K.-C. Chou, Z.-B. Su, B.-L. Hao, and L. Yu, Equilibrium and nonequilibrium formalisms made unified, Phys. Rep. 118, 1 (1985).

[40] R. P. Feynman and F. L. Vernon, Jr., The theory of a general quantum system interacting with a linear dissipative system, Ann. Phys. (N.Y.) 24, 118 (1963).

[41] J. Gasser and H. Leutwyler, Chiral perturbation theory to one loop, Ann. Phys. (N.Y.) 158, 142 (1984).

[42] S. Weinberg, The Quantum Theory of Fields, Vol. 2 (Cambridge University Press, Cambridge, England, 1996). 
[43] M. Kardar, G. Parisi, and Y.-C. Zhang, Dynamic Scaling of Growing Interfaces, Phys. Rev. Lett. 56, 889 (1986).

[44] L. M. Sieberer, A. Chiocchetta, A. Gambassi, U. C. Täuber, and S. Diehl, Thermodynamic equilibrium as a symmetry of the Schwinger-Keldysh action, Phys. Rev. B 92, 134307 (2015).
[45] C. Aron, G. Biroli, and L. F. Cugliandolo, (Non) equilibrium dynamics: A (broken) symmetry of the Keldysh generating functional, SciPost Phys. 4, 008 (2018).

[46] Y. Kuramoto, Chemical Oscillations, Waves, and Turbulence, Springer Series in Synergetics (Springer, Berlin, 2012).

[47] H. Mori, G. C. Paquette, and Y. Kuramoto, Dissipative Structures and Chaos (Springer, Berlin, 2013). 